\title{
An efficient and pragmatic approach for regulatory aquatic mixture risk assessment of pesticides
}

\author{
Christian Dietrich ${ }^{1}$, Magnus Wang ${ }^{1}$, Markus Ebeling ${ }^{2}$ and Anja Gladbach² ${ }^{*}$ (D)
}

\begin{abstract}
The current regulatory approach to address aquatic mixture toxicity for pesticides in the EU (EFSA J 11:3290, 2013) is rather complex: in typical cases it requires conducting the entire mixture risk assessment scheme for every exposure scenario separately (e.g. 6-8 ecotoxicological endpoints, for each of the nine exposure scenarios for the European Central Zone with 24 common mitigation measure combinations result in over 1700 sub-scenarios to be assessed). This article discusses the available concepts for a mixture toxicity assessment, the key questions raised and the implementation of these questions in existing risk assessment approaches. Based on this, an alternative, more efficient assessment scheme for aquatic mixture risk assessment (AMiRA) is proposed with the aim of facilitating the practical conduct and interpretation of the assessment while addressing the key questions and preserving the level of protection. The scheme assesses product risk (including a check for non-additive effects), the presence of a risk driver and gains efficiency by the straightforward use of risk quotients $(R Q)$ to calculate mixture risk quotients $\left(R Q_{\text {mix }}\right)$ that are equivalent to the calculation of exposure toxicity ratios for a mixture $\left(E_{T R} R_{\text {mix,CA}}=\right.$ Exposure-Toxicity-Ratio for mixtures based on concentration addition) proposed by EFSA (EFSA J 11:3290, 2013). A case study is provided underlining the equivalence of the proposed approach to the EFSA (EFSA J 11:3290, 2013) decision tree.
\end{abstract}

\section{Background}

In the regulation of plant protection products (PPP), risk assessments are a key requirement to ensure human and environmental safety. In these risk assessments hazard and exposure for the active substances are addressed. However, PPP are sometimes formulated and applied as mixture products, in which two or more active substances are combined to, e.g. increase the efficacy to a broader range of pest organisms or for resistance management. Thus, in recent years the assessment of mixture toxicity (i.e. toxicity from application of a product with multiple constituents, not from e.g. spray series) has increasingly gained interest, resulting in the development

\footnotetext{
*Correspondence: anja.gladbach@bayer.com

2 Bayer AG, Crop Science Division, Alfred-Nobel-Str. 50, 40789 Monheim am Rhein, Germany

Full list of author information is available at the end of the article
}

of conceptual frameworks and guidance documents addressing the mixture toxicity risk assessment for human health and the environment $[3,4,11,24,25$, 27-29]. In theory, mixture effects from pesticides can be expected for all environmental situations in which active substances co-occur resulting in a multitude of potential mixture compositions to be assessed for their risk, so some filter needs to be applied to get to mixtures of concern. Active substances which are most likely to co-occur and occur at the highest relative concentrations are those formulated and applied together.

In Europe, mixture risk is addressed in new legal requirements to consider potential interactions between active substances (a.s.) that triggered the development of mixture risk assessment approaches. Regulation (EC) No. 1107/2009, Article 29, states that “...] interaction between the active substance, safeners, synergists and coformulants shall be taken into account in the evaluation

\section{Springer Open}

(c) The Author(s) 2022. Open Access This article is licensed under a Creative Commons Attribution 4.0 International License, which permits use, sharing, adaptation, distribution and reproduction in any medium or format, as long as you give appropriate credit to the original author(s) and the source, provide a link to the Creative Commons licence, and indicate if changes were made. The images or other third party material in this article are included in the article's Creative Commons licence, unless indicated otherwise in a credit line to the material. If material is not included in the article's Creative Commons licence and your intended use is not permitted by statutory regulation or exceeds the permitted use, you will need to obtain permission directly from the copyright holder. To view a copy of this licence, visit http://creativecommons.org/licenses/by/4.0/. 
of plant protection products." For an environmental risk assessment, the highest exposure and consequent risk is to be expected at the edge-of-field for aquatic organisms. Thus, a first focus area was the integration of mixture risk assessments in aquatic risk assessments [2, 18, 22, 28].

A basic concept of the mixture assessment is to use endpoints from existing studies in order to avoid unnecessary testing, for both animal welfare reasons and efficiency regarding the preparation and review of regulatory submissions. Given the multitude of potential mixture compositions (even after identifying for mixtures of concern) due to, e.g. different environmental behaviour of active substances in a mixture toxicity is increasingly addressed by toxicity calculations based on established concepts like Concentration Addition (CA) or Independent Action (IA) rather than the generation of new data. Key concepts of all mixture risk assessment approaches are to assess the toxicity of mixtures based on CA, IA or other assumptions, the existence of a risk driver or the proportion of the substances in a mixture vs. the proportion in the environment $[2,18,22,28]$.

The regulatory approach to address aquatic mixture risk of pesticides in the $\mathrm{EU}$, i.e. the guidance on tiered risk assessment for plant protection products (PPP) for aquatic organisms in edge-of-field surface waters [14], combines several of these concepts. Large parts of EFSAs approach are based on previous publications by EFSA, namely the guidance on birds and mammals [13] and the scientific opinion on the science behind the development of a risk assessment of PPPs on bees [12]. The previously published approaches were adapted by EFSA [14] considering the specific characteristics of aquatic risk assessment by relying on review literature $[2,22,28]$.

The approach proposed by EFSA [14], however, is a rather complex 10-step decision tree including the comparison of measured vs calculated product toxicity (and the subsequent use of either one depending on the comparison outcome), the check for a toxicity driver and potential synergism or antagonism. Although this decision tree captures all relevant aspects and questions in aquatic mixture risk assessment, opportunities to focus the risk assessment on the most critical scenarios exist but are often not implemented in regulatory practice. One key aspect here is the sentence: "Note, this mixture RA scheme has to be carried out for each endpoint and exposure scenario separately unless it is evident that one specific endpoint/exposure scenario combination clearly drives the risk." (EFSA [14], point 2.5). For aquatic risk assessments in EU legislation, exposure is estimated based on FOCUS [16], a scenario-based, stepwise procedure for exposure in edge-of-field surface waters. While Step 1 and 2 result in one or two worst-case exposure scenarios across EU, step 3 already considers numerous different scenarios across EU (ponds, ditches, streams, e.g. 9 for the central zone) and step 4 allows taking into account matrixes of exposure reduction measures (drift and run-off buffers of different widths combined with drift reduction between 50 and 90\%) to identify measures needed to ensure acceptable risk for the use under evaluation. Depending on the risk profile of the active substance, different FOCUS steps might be needed to conclude a risk assessment and for the different active substances in a mixture, the most sensitive aquatic taxa can be different. With this combination of FOCUS steps and 6-8 eco-endpoints (acute/chronic for different taxa), the scenario and eco endpoint specific mixture risk assessment can result in hundreds of pages of complex calculations and the identification of a worst-case scenario is not necessarily straightforward for a mixture product.

Tools have been designed to address the complex calculations as per the aquatic guidance decision tree and make them easier to conduct and implement in a regulatory submission and assessment (e.g. [9, 19]). However, these tools only facilitate the conduct of the multiple calculations, but do not remove the complexity of the required assessments in terms of numerous scenarios to be addressed and the associated efforts in interpretation of the results and conclusions on calculated risks and management needs.

Recently, Chen et al. [8] proposed a framework for risk assessment for pesticide mixtures on aquatic ecosystems in China, aiming to reduce unnecessary complexity and workload by introducing a screening stage prior to a risk assessment scheme similar to the approach by EFSA [14]. Furthermore, elaborated developments regarding tiered assessment schemes took place in Europe to improve concepts from existing risk assessment schemes (e.g. the identification of risk or toxicity drivers and the use of alternative methods for calculating mixture toxicity [23]. However, the shortcoming of not removing complexity is even acknowledged in the tools referred to above e.g. regarding the Toxic Unit (TU) approach recommended in EFSA [14] stating that this "seems to add rather more complexity than facilitating the MixTox assessment (as initially intended)". Any simplification whilst maintaining the level of protection would be of benefit. Only few risk assessment schemes have been proposed so far specifically aiming to clearly reduce the complexity of the approach by EFSA [14] while preserving its level of protection $[10,31]$.

In the present study, the key questions raised in a mixture risk assessment and the implementation of these questions in existing risk assessment approaches for aquatic mixture risk assessment under Regulation (EC) $1107 / 2009$ are discussed (whilst as a general approach, it 
may well be applicable elsewhere). Finally, an alternative simplified approach for aquatic mixture risk assessment (AMiRA) is developed with the aim to facilitate the practical conduct of a risk assessment while answering the key questions and preserving the level of protection.

\section{Key questions for a mixture risk assessment}

For mixture risk assessments the following points need to be considered: (1) concepts regarding the combination toxicity of substances (e.g. interaction or applicability of CA and IA); (2) risk assessment calculation for predicted mixture toxicity (ETR vs. RQ); (3) consideration of a worst-case exposure scenario; (4) identification of substances which drive the exposure or risk assessment and (5) use of measured or calculated mixture toxicity.

The first two points have been broadly discussed in the scientific literature. Therefore, detailed information about these two points is provided in the supplemental material S1. The remaining three points have not yet been discussed in detail. Therefore, these points are outlined in detail in the following sections.

\section{Identifying a regulatory worst-case exposure scenario}

As outlined above, European regulation requires capturing the variability in exposure resulting from different soil, climate and other conditions for the risk assessment of pesticides [16]. In Europe, a standard set of nine surface water exposure scenarios is used for the risk assessment [1], with an increasing number of scenarios to be assessed when the consideration of mitigation measures at FOCUS steps $3 / 4$ is necessary to show an acceptable risk from a use: for each of the nine pond, ditch and stream scenarios there are 28 potential mitigation measure combinations from different spray buffers, vegetation strips and nozzle reductions, resulting in a total of 252 sub-scenarios to be assessed for each considered aquatic toxicity endpoint. However, depending on the risk profile of substances, risk mitigation options might only be necessary for a subset of scenarios and/or taxa.

Given the tiered approach of FOCUS (with lower steps being more conservative) and the fact that for the risk assessment of individual active substances calculations will only be conducted to the level needed to pass the risk assessment, the individual active substances within a mixture may not necessarily have reached equivalent steps in the FOCUS evaluation.

Since the predicted environmental concentrations (PECs) of individual active substances are different in each of the scenarios a fundamental question in the mixture risk assessment is, whether it is possible to define a worst-case mixture exposure scenario out of these based on PECs of individual substances or whether a worstcase can only be defined for the risk assessment when the composition and subsequent potential toxicity of mixtures associated with each scenario are taken into account.

One option is to conduct the risk assessment for every single scenario separately and to select the worst-case risk assessment afterwards. Another option is to search for a worst-case scenario among the candidate scenarios up front, just selecting the scenario with the worst-case exposure in total (i.e. the scenario with the highest sum of PECs of individual substances) or an artificial worstcase scenario consisting of each worst-case PECs from multiple scenarios [8]. Although the latter approach clearly results in a (likely overly conservative) worst-case exposure scenario, both approaches neglect the specific toxicity of the individual substances in the mixture. An advanced alternative is a scenario selection based on worst-case risk quotients (RQs). Risk quotients are defined as the ratio between the exposure (i.e. PECs) and a toxicological endpoint (i.e. ECx) including an assessment factor (AF) defined in e.g. the aquatic guidance document by EFSA [14]:

$$
\mathrm{RQ}=\frac{\mathrm{PEC}}{\mathrm{ECx} / \mathrm{AF}} .
$$

The quotient ECx/AF corresponds to the regulatory acceptable concentration (RAC) or to the predicted noeffect concentration (PNEC).

A combined risk quotient from the mixture $R Q_{\text {mix }}$ can be obtained by summing up the RQs from the single substances $\left(R_{\text {mix }}=\sum R Q\right)$. This combined risk quotient can be used to select a worst-case risk scenario.

To illustrate the worst-case character of a scenario selection based on $\mathrm{RQ}_{\text {mix }}$, an example is shown in Table 1 . It can be seen that the $R Q_{\text {mix }}$ calculation is equivalent to the calculation of ETR $\mathrm{mix}_{\mathrm{CA}}$ values based on the assumption of concentration addition (CA) that are used by EFSA [14] to calculate mixture toxicity. The worstcase scenario indicated by the highest $\mathrm{RQ}_{\text {mix }}$ therefore corresponds to the worst-case risk assessment from all scenarios.

Hence, in conclusion, it has been illustrated that a scenario selection based on the worst-case $R Q_{\text {mix }}$ corresponds to the worst-case risk assessment from all scenarios when CA-based mixture risk has been calculated.

In summary, there are three main reasons for using (mixture) risk quotients in the context of scenario selection for the risk assessment:

1) Risk quotients closely relate to the idea of the risk assessment itself, providing an immediate and intuitive understanding of (un)acceptability of a risk 
Table 1 Example with toxicity and exposure values for hypothetical substances and scenarios based on worst-case $R Q_{\text {mix }}$

\begin{tabular}{|c|c|c|c|c|c|c|}
\hline \multirow{4}{*}{$\begin{array}{l}\text { EC50 [mg a.s./L] } \\
\text { RAC [mg a.s./L] } \\
\text { Scenario }\end{array}$} & \multirow{2}{*}{\multicolumn{2}{|c|}{$\frac{\text { Substance A }}{1.1}$}} & \multicolumn{2}{|l|}{ Substance B } & \multicolumn{2}{|c|}{ Mixture risk } \\
\hline & & & \multicolumn{4}{|l|}{82} \\
\hline & \multicolumn{2}{|l|}{0.011} & \multicolumn{4}{|l|}{0.82} \\
\hline & PEC [mg a.s./L] & $\mathrm{RQ}$ & PEC [mg a.s./L] & $\mathrm{RQ}$ & $\mathrm{RQ}_{\text {mix }}$ & $A F^{*} E^{\prime} R_{\text {mix }, C A}$ \\
\hline 1 & 0.001 & 0.091 & 0.317 & 0.387 & 0.477 & 0.477 \\
\hline 2 & 0.002 & 0.182 & 0.291 & 0.355 & 0.537 & 0.537 \\
\hline 3 & 0.007 & 0.636 & 0.211 & 0.257 & 0.894 & 0.894 \\
\hline 4 & 0.006 & 0.545 & 0.256 & 0.312 & 0.858 & 0.858 \\
\hline $5^{b}$ & 0.008 & 0.727 & 0.195 & 0.238 & 0.965 & 0.965 \\
\hline
\end{tabular}

a The calculated $R Q_{\text {mix }}$ is identical to the $C A$-based $E T R_{\text {mix, } C A}$ multiplied by the assessment factor $(A F=100)$

${ }^{\mathrm{b}}$ Selected worst-case scenario

without additional information necessary on AF or acceptability criteria.

2) The use of mixture risk quotients allows ranking of and an identification of a worst-case risk assessment.

3) Risk quotient calculation is part of the aquatic risk assessment for individual active substances and after the selection of a worst-case scenario the individual substance RQs can directly be summed up and used for a risk assessment of the mixture (instead of investing in additional calculations of scenario-specific PECmix and ETRmix values, which are mathematically equivalent as shown in the Additional file 1: S1).

\section{Identification of substances which drive the exposure or risk assessment}

In a mixture of chemicals some chemicals will contribute more to the mixture toxicity than others. Usually, only a handful of substances contribute to the vast majority of the mixture toxicity $[15,21,30]$. If one substance is the main contributor to the overall toxicity of a mixture (usually attributed to a $\geq 90 \%$ contribution, e.g. EFSA [14]), it may be sufficient to conduct the risk assessment only for that one substance.

Brown [7] demonstrated the estimation of the toxicity of a mixture by adding up proportional toxicities obtained from the ratio between the concentration of a mixture component and its toxicological endpoint:

$$
\mathrm{TU}_{i}=\frac{c_{i}}{\mathrm{ECx}_{i}} .
$$

The ratio between single proportional toxicities (socalled 'toxic units', TU) and their sum are used to quantify the magnitude of toxicity contribution [15, 21, 30]. Notably, the concept of proportional toxicities and their summation correspond to the concentration addition concept for calculating mixture toxicity, i.e. the concepts are transferrable.

If the identified toxicity driver in a product $(=$ fixed composition) is only present in a low proportional concentration in an environmental mixture, it may actually not drive the environmental risk; other, less toxic components are risk drivers due to proportional high concentrations in the environment. These differences between concentrations in a product and environmental concentrations of active substances may arise based on the different environmental fate and transport after emission. Hence, from the perspective of a risk assessor, a risk driver would be more appropriate than the toxicity driver obtained from the evaluation of TUs.

An alternative approach could therefore be based on proportional risk rather than on proportional toxicity. A proportional risk quotient (PRQ) could simply be calculated by applying the concept used for $\mathrm{TU}$ to the ratio between RQs from single substances and the overall $\mathrm{RQ}_{\text {mix }}$ :

$$
\mathrm{PRQ}_{i}=\frac{\mathrm{RQ}_{i}}{\mathrm{RQ}_{\text {mix }}}
$$

A risk driver could then be identified if one substance clearly drives the risk from the mixture, i.e. if the maximum RQ from one active substance represents the vast majority of the $\mathrm{RQ}_{\text {mix }}$ (similar to the maximum cumulative ratio approach by [26]. This approach is only valid if no synergism has been detected.

To quantify the deviation between measured and calculated mixture toxicity, the model deviation ratio (MDR) has been introduced [5]. It is calculated by dividing the calculated mixture toxicity by the observed mixture toxicity. If the MDR markedly differs from 1 , the calculated mixture toxicity contradicts the measured mixture toxicity. Potential antagonism is identified for small MDRs $(<0.2)$ and potential synergism 
is identified for high MDRs ( $>5)$. In case of apparent antagonism $(\mathrm{MDR}<0.2)$ the calculated mixture risk exceeds the measured mixture risk at least by a factor of five, meaning that the risk from a single risk driving substance would exceed the measured mixture risk at least by a factor of $4.5(90 \% * 5)$. Hence, the PRQ calculations are considered to be conservative for apparent antagonism. As depending on the toxicity profile of substances sensitivity differences for different taxonomic groups might be larger or smaller, an assessment for a risk driver must be carried out for each taxonomic group separately.

In the current EFSA [14] decision tree, it is not clearly stated whether the composition of a mixture refers to the product composition or the environmental concentrations, i.e. if the assessment is identifying a toxicity or risk driver. Consequently, depending on the interpretation of the term "composition" different assessments for either a toxicity or risk driver lead to different conclusions in the decision tree. For instance, a calculation tool for EFSA [14] developed and released by FPS Health, Food Chain Safety and Environment for Belgium [17], considers the product composition to derive TU and identifies a toxicity driver; whereas an alternative calculation tool developed as an initiative of regulators from different European member states [19] considers environmental concentrations and consequently derives a risk driver. The FAQ accompanying the latter calculation tool highlights the following: "Note also that the driver is often based on the product composition (as stated in the guidance) but this is scientifically not correct (as clarified in an email exchange with EFSA). The tool (assessment Step 5-Driver assessment) can be used to check if this is relevant in the specific case (i.e. if the results of Eq. 14 are different in case the proportions are based on the composition of the product instead of the composition at the PECmix the latter of which may lead to different results for the FOCUS scenarios under assessment)". This clarification of the influence of the composition used (product vs environment) and the impact on the conclusion on a toxicity or risk driver is a very important addition. With this additional clarification, the here proposed PRQ is in line with the assessment intended in the EFSA [14] decision tree.

\section{Using measured or calculated mixture toxicity}

When measured mixture toxicity data are missing, calculated mixture toxicity based on component-based approaches are considered the second best option [18]. In order to not increase testing requirements, the conceptual use of calculated mixture toxicity is widely considered to be acceptable [14, 22].
If measured data are available, there are essential questions arising from the use of these data:

1) Does the calculated mixture toxicity differ from the results from the measured data (and if yes, do measured data show higher or lower toxicity than the calculated toxicity and what are potential reasons for this)?

2) Are the results from laboratory tests representative for the application of the mixture under field conditions (i.e. does the formulation composition in the toxicological tests reflect the mixture composition of the substances in the field and is information on ecologically relevant metabolites available from formulation testing)?

3) If required: Do substances other than active substances need to be considered in the mixture risk assessment or overall exposure levels (e.g. metabolites and or other formulation components)?

Belden et al. [5] observed that $88 \%$ of the reviewed experiments evaluating the CA model (regardless of MoA and pesticide target groups) had MDRs ranging between 0.5 and 2.0 (about 5\% of MDRs were less than 0.5 and about $5 \%$ were greater than 2.0). According to EFSA [14] a deviation factor of five $(0.2 \leq \mathrm{MDR} \leq 5)$ is considered to be acceptable to account for biological and other variation in testing.

Whether the results from laboratory tests are representative for the application of the mixture under field conditions can be evaluated by comparing the calculated mixture toxicity based on (i) the mixture composition as used in the toxicological tests and (ii) the mixture composition as expected from realistic exposure scenarios (i.e. using PECs from FOCUS surface water scenarios). However, although this comparison of mixture composition is an important point, in the absence of synergy (as determined by MDR) it is an unnecessary complication for the risk assessment and protection will be ensured by a CA approach. The allowed magnitude of deviation has then to be based on expert judgement (e.g. maximum deviation of $20 \%$ as proposed by EFSA [14] and chen et al. [8].

It is generally advised to check whether metabolites of toxicological relevance have to be included into the mixture risk assessment exposure level [14]. Although metabolites are in most cases less toxic than the respective parent substances the risk from exposure to them is assessed by calculating metabolite PECs. With both toxicity and exposure information available, they thus can be included in a quantitative CA mixture risk assessment if they are identified as contributing significantly to the risk. However, typically there is little information about 
metabolite concentrations available from a toxicity study with a specific formulation because usually only the concentrations of the active substances within the tested product are confirmed analytically. In addition, the exposure differences between specific FOCUS surface water scenarios (especially between pond, ditch and stream scenarios) can be very pronounced in practice. Therefore, it is difficult to compare the toxicity of the product based on formulation composition and the toxicity of the product based on PECs under field conditions and the usability of measured data are usually very limited. Exposure by spray drift best represents the conditions of both the aquatic test system and in the environment after entry of a product into the waterbody, as here no environmental degradation and (re)distribution processes affect the composition of ingredients. Since the mixture risk assessment according to EFSA [14] should only be based on measured mixture toxicity data if the mixture composition in the tested product in laboratory tests is similar to the mixture composition at the $\mathrm{PEC}_{\text {mix }}$ in terms of the relative proportions of the individual a.s., and with metabolites not being present in the initial product composition, only calculated mixture toxicity can take into account both active substances and relevant metabolites. It thus can be argued to always use calculated mixture toxicity for the mixture risk assessment and use measured data within a risk assessment for the product after spray-drift entry.

\section{Risk assessment approaches by EFSA [14] and Chen et al. [8]}

The guidance on tiered risk assessment for plant protection products for aquatic organisms in edge-of-field surface waters by EFSA [14] is the most relevant approach to address aquatic mixture risk for pesticides in the EU, this approach is discussed in detail in the following section. Chen et al. [8] recently proposed a framework for risk assessment for pesticide mixtures on aquatic ecosystems in China similar to EFSA [14] but including a screening stage, this approach is thus considered as well.

\section{Risk assessment scheme by EFSA [14]}

The risk assessment by EFSA [14] is based on the concept of $\mathrm{CA}$ and an approach which initially includes:

1) The detection of potential antagonism/synergism by calculating the model deviation ratio $(\mathrm{MDR}=\mathrm{calcu}-$ lated mixture toxicity/measured mixture toxicity).

2) A check for 'toxicological similarity' by comparison of calculated $\mathrm{ECx}_{\text {mix }}$ values based on (i) formulation composition and (ii) PEC proportions.

3) A check for toxicity driving single active substances.
Following this initial review, the decision scheme by EFSA [14] can result in three types of risk assessment:

1) The risk assessment could be based on calculated mixture toxicity if (i) measured mixture toxicity data are not available (Step 1) and no synergy is demonstrated or anticipated (Step 7) or (ii) if uncertainty over the measured data (e.g. unexplained antagonism, Step 9) remains or if (iii) the measured mixture composition is not toxicologically similar to the mixture composition at $\mathrm{PEC}_{\text {mix }}$ (Step 3) and no toxicity driver could be identified (Step 5).

2) The risk assessment could be based on measured mixture toxicity if the measured and calculated mixture toxicity differ by less than a factor of five $(0.2 \leq \mathrm{MDR} \leq 5$, Step 2$)$ and the measured mixture composition is similar to the mixture composition at $\mathrm{PEC}_{\text {mix }}$ (Step 3).

3) The risk assessment could be based on a single active substances if it clearly drives the mixture toxicity (Step 5).

Measured mixture toxicity data are not strictly required to conduct the risk assessment, but have to be taken into account if there is evidence for synergistic interaction from the comparison of the CA model against existing measured data. An overview about the different steps is given in Fig. 1. Note that the check for the apparent synergism (step 10 in EFSA, [14]) is missing in the flowchart, because without exception it always leads to the same subsequent step (step 3 in EFSA [14]).

Overall, there are many branches and decisions within the decision tree all leading to the final evaluation of ETRs based on measured or calculated mixture toxicity or single active substances.

Notably, the opportunity to use $\mathrm{RQ}_{\text {mix }}$ instead of $\mathrm{ETR}_{\text {mix-CA }}$ for the risk assessment is offered in EFSA [14] for single species mixture risk assessments but is only linked to the case of different AFs although the endpoints to be used for the mixture risk assessment refer to the same taxonomic group. However, in the associated calculation tool, $\mathrm{RQ}_{\text {mix }}$ is offered not just when AFs differ but also when data of different species other than standard tier 1 are used. This highlights the additional advantage of the RQ metric enabling a direct comparison across different tiers of a risk assessment which is not possible with $\mathrm{ETR}_{\text {mix }}$. Based on the fact that CA has been developed and evaluated for tier 1 standard laboratory data, more research is needed to evaluate whether $R_{\text {mix }}$ calculations are also robust when including higher-tier toxicity data. Therefore, in principle, $R Q_{\text {mix }}$ could replace ETR $_{\text {mix }}$ for all situations where tier 1 standard data are used in the risk assessment since it has been shown for 


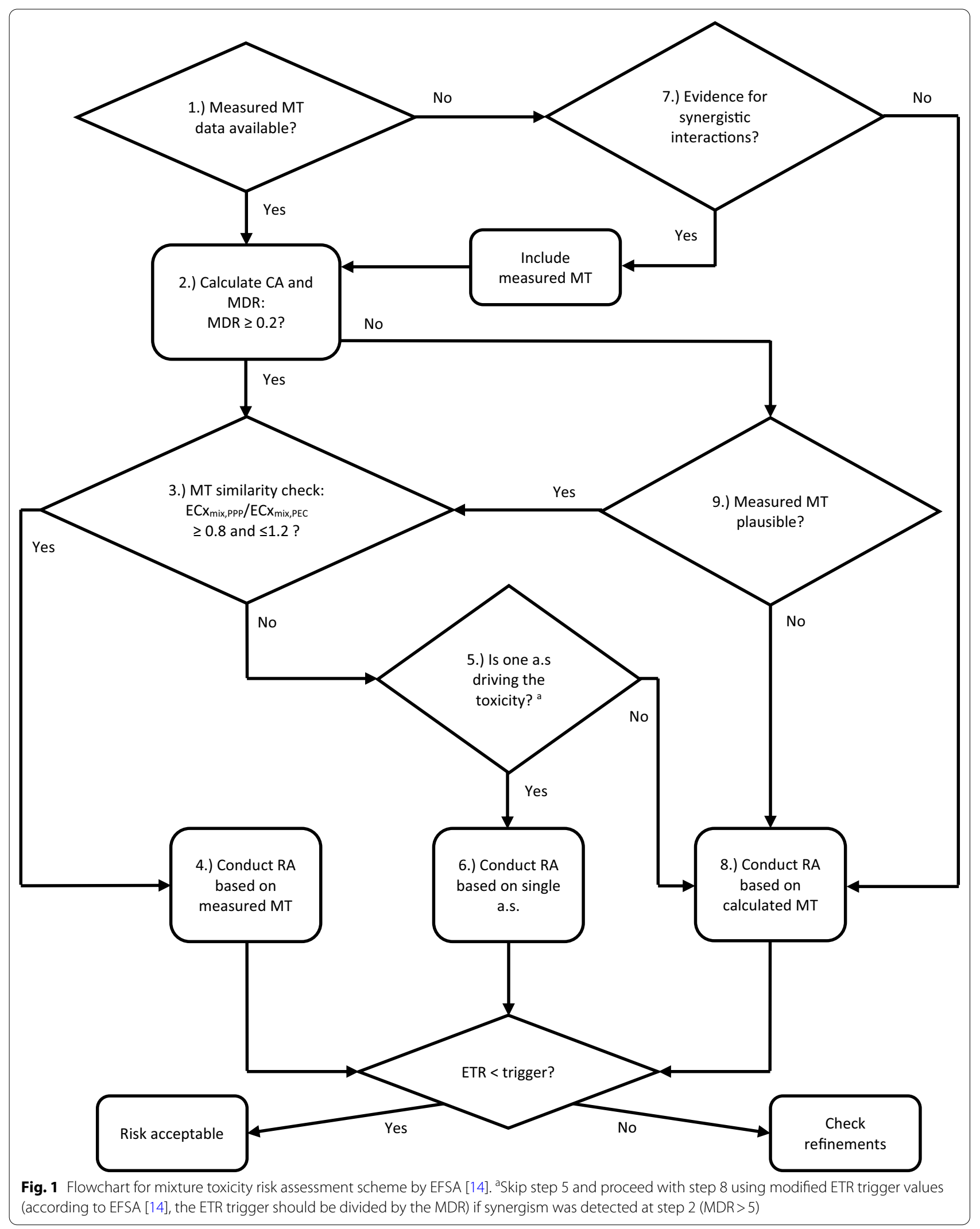


both approaches that they are equivalent. There are some points limiting the efficiency of the approach by EFSA [14] outlined in the following:

1) There should be no need to check for toxicity drivers (step 5 in EFSA, [14]) after interaction (i.e. antagonism/synergism) was detected. In general, this step would be more appropriate prior to the mixture toxicity risk assessment to enhance an efficient risk assessment (currently, the check for a risk/toxicity driver is conducted regardless of the magnitude of the overall risk). Furthermore, the use of a risk driver would be a preferable alternative to the use of a toxicity driver in order to also consider the exposure level and thus to increase the level of protection.

2) The absence of measured data requires a decision whether synergistic effects are evident (step 7 in EFSA, [14]). In the absence of measured data allowing an MDR calculation, this could include any relevant toxicological information including read across from other species and chemicals based on mode of action or TK/TD information on the mixture. If apparent synergism is detected $(M D R>5)$, the scheme suggests that the ETR trigger is divided by the MDR to account for this is the assessment. However, if the mixture ratio tested was similar to that being assessed, the measured toxicity could be used directly rather than applying an additional $\mathrm{AF}$ and basing the assessment on calculated mixture toxicity

3) Step 3 in EFSA [14] is used to check whether measured or calculated mixture toxicity data could/ should be used for the risk assessment. It does so by comparing measured toxicity data for the product $\left(\mathrm{ECx}_{\text {prod }}\right)$ to calculated $\mathrm{ECx}_{\text {mix }}$ values based on individual substance toxicity and environmental exposure. Based on the decision tree provided in EFSA [14] in addition to proceeding from Step 2 to Step 3, it is also possible to return to Step 3 from Steps 9 and 10 (recheck for antagonism/synergism). As steps 9 and 10 are reached after Step 2 (=MDR analysis showing that the measured mixture toxicity deviates much from the calculated one) this duplicates the work and could be overcome by directly continuing to work with the calculated toxicity. Furthermore, another inconsistency can be seen at step 10 in EFSA ([14], recheck for synergism): It is only possible to reach Step 10, when measured data are used. However, the question regarding measured data are again raised in this step although it has already been answered (when no measured data are available, one should proceed with Step 8, where the risk assessment is based on calculated mixture toxicity). Hence,
Step 10 can generally be skipped and one can directly proceed with Step 3.

4) The approach by EFSA [14] requires conducting the entire risk assessment scheme for every exposure scenario separately (e.g. six to eight toxicological endpoints, depending on the PPP type, for each of e.g. nine exposure scenarios for the European Central Zone with 24 common mitigation measure combinations result in over 1700 sub-scenarios to be assessed).

\section{Risk assessment scheme by Chen et al. [8]}

In general, the approach by Chen et al. [8] can be considered an extended variation of the approach by EFSA [14] using some of EFSA's concepts (e.g. the calculation of MDR and the check for toxic similarity are essentially similar). Chen et al. [8] aimed to reduce the workload for a mixture toxicity assessment by introducing a preliminary screening stage (tier 0 ) and adjusted widely accepted scientific concepts (e.g. the concept of concentration addition) to local needs (e.g. use of local scenarios). The tier 0 risk assessment is a screening stage focussing on single a.s. analyses. Risk quotients (RQs) of single a.s. are calculated by dividing the PEC of the a.s. by the respective PNEC ( $\mathrm{PNEC}=\mathrm{EC} 50_{\text {mix }}$ /uncertainty factor). Measured and calculated mixture toxicity are not relevant at this stage and the entire mixture toxicity risk assessment can be omitted by prior analysis of single active substances. The risk assessment has to be conducted for the taxonomic groups of aquatic vertebrates (i.e. fish), aquatic invertebrates (i.e. daphnids), and primary producers (i.e. green algae). Chen et al. [8] propose the use of worst-case PECs from all exposure scenarios to create one worst-case exposure scenario, subsequently leading to (potentially overly conservative) worst-case RQs.

The use of a worst-case exposure scenario is based on the concept of a "risk envelope approach", an approach commonly used in environmental risk assessments to cover less critical scenarios by conducting a risk assessment for the identified worst-case scenario. The risk envelope thus yields intrinsic conservatism for all scenarios less critical than the worst-case, allows to focus the risk assessment on the most sensitive scenario and/ or organism and therefore can dramatically reduce the workload negating the need for assessing (and regulatory review of) less critical scenarios.

Additionally, potential refinements (e.g. PEC refinements) are conducted prior to mixture toxicity analyses at tier 1 of the risk assessment scheme. While measured mixture toxicity is not needed at tier 0 in Chen et al. [8], it becomes mandatory when entering tier 1 . 
Tier 1 from Chen et al. [8] is then generally similar to the approach by EFSA [14]: concentration addition (CA) is also used as default assumption for calculated mixture toxicity, the 'toxicological similarity' (TS) corresponds to step 3 from EFSA [14] and Chen et al. [8] propose the same thresholds for TS and MDR analyses. For the final risk assessment, Chen et al. [8] based the calculations on the use of RQs. They also point out the use of $R_{Q_{\text {mix }}}$ as a "pragmatic approach adopted by EFSA where the sum of risk quotients generated with data at different tiers can also be used in this framework." Finally, Chen et al. [8] do not evaluate any toxicity driver or risk driver.

\section{Comparison of approaches}

The approaches by EFSA [14] and Chen et al. [8] share some of the concepts applied in a mixture risk assessment: both approaches rely on the concept of concentration addition for calculating mixture toxicity (applying the concept of independent action is an option if its conditions are fulfilled, e.g. if there is a dissimilar MoA) and both approaches rely on the concepts of TS and the MDR. The approaches differ in terms of their selected exposure scenarios, their need for measured mixture toxicity data, their toxicity driver assessment, and their calculation basis for the risk assessment (ETR vs. RQ). A comparison of the mixture risk assessment components applied by EFSA [14] and Chen et al. [8] is provided in Table 2. Notably, EFSA [14] proposed a simplified approach for mixture risk assessment (Sect. 10.3.7 in original publication) similar to the tier 0 screening stage by Chen et al. [8]. However, this simplified approach is not included in the EFSA [14] mixture risk assessment decision scheme.

\section{Conclusions from the theoretical comparison of approaches}

The aquatic mixture toxicity risk assessment scheme by EFSA [14] is the first regulatory approach in Europe addressing many of the key questions for a mixture toxicity risk assessment. Both EFSA [14] and Chen et al. [8] are based on the same well-established scientific concepts. Overall, both approaches apply similar levels of conservativeness to the risk assessment as other risk assessment schemes for single substance risk assessments (e.g. screening levels with high conservatism in exposure and effect, use of assessment factors to cover for remaining uncertainties). The mixture risk assessment scheme by EFSA [14], however, still faces some inconsistencies and conceptual weaknesses (e.g. the missing clarity regarding the use of toxicity drivers or of risk drivers). In addition, the approach causes a considerable workload for conducting a risk assessment for all taxonomic groups and exposure scenarios (over 1700 potential scenarios have to be assessed) and also increases the time for evaluation and to draw a conclusion for risk assessment and management. Please note that by skipping ETR $_{\text {mix }}$ and directly using the $\mathrm{RQ}_{\text {mix }}$ approach, advocated in EFSA [14], the assessment can be easily simplified.

Chen et al. [8] extended the mixture toxicity risk assessment by a useful screening stage in order to minimise the workload for risk assessors, but without considering the potential occurrence of risk drivers.

Therefore, an alternative pragmatic approach is developed in the following. It is designed to enable efficient risk assessment, reducing the work necessary to for low risk formulations and scenarios and focussing on the more critical areas of the risk assessment.

Table 2 Comparison of mixture risk assessment components in EFSA [13] and Chen et al. [8]

\begin{tabular}{|c|c|c|}
\hline Mixture risk assessment components & EFSA [14] & Chen et al. [8] \\
\hline Screening stage & $\begin{array}{l}\text { No screening stage included in the decision } \\
\text { scheme }\end{array}$ & $\begin{array}{l}\text { Tier } 0 \text { screening stage focussing on single a.s. } \\
\text { analyses }\end{array}$ \\
\hline Selection of suitable exposure scenario & $\begin{array}{l}\text { Required to conduct the entire risk assessment } \\
\text { scheme for every exposure scenario separately }\end{array}$ & $\begin{array}{l}\text { Use of worst-case PECs from all exposure sce- } \\
\text { narios to create one worst-case exposure scenario }\end{array}$ \\
\hline $\begin{array}{l}\text { Identification of substances which drive the } \\
\text { exposure or risk assessment }\end{array}$ & $\begin{array}{l}\text { Toxicity driver approach using the concept of } \\
\text { TUs }\end{array}$ & No toxicity or risk driver evaluated \\
\hline Measured mixture toxicity data & Not strictly required & Required after screening stage \\
\hline $\begin{array}{l}\text { Default assumption for calculated mixture } \\
\text { toxicity }\end{array}$ & CA (use of IA possible under certain conditions) & CA (use of IA possible under certain conditions) \\
\hline $\begin{array}{l}\text { Comparison of measured and calculated mixture } \\
\text { toxicity }\end{array}$ & $\begin{array}{l}\text { MDR (not required if no measured data avail- } \\
\text { able) }\end{array}$ & MDR (required after screening stage) \\
\hline Risk assessment & $\begin{array}{l}\text { Based on ETR (use of RQ possible under certain } \\
\text { conditions) }\end{array}$ & Based on $\mathrm{RQ}$ \\
\hline
\end{tabular}




\section{Development of an alternative approach for the mixture risk assessment for plant protection products for aquatic organisms Key points for the development of the approach}

A mixture toxicity risk assessment following currently available assessment schemes can be very complex and time-consuming. The purpose of the here presented AMiRA approach is to reduce the required workload for such an assessment while preserving the current high level of protection of the environment. Like the approaches for calculating mixture toxicity by EFSA [14] and Chen et al. [8], AMiRA is intended to be used under the assumption of CA (after checking for interactions between substances indicating deviation from CA, applying IA is an option, e.g. if there is a dissimilar MoA or if effects at lower doses are considered).

A schematic overview of the proposed AMIRA scheme is shown in Fig. 2.

Key points considered to gain efficiency are:

1) Assess whether available measured data show any deviation from CA and use measured data in a product risk assessment for spray-drift entry (AMiRA Step 1 and 2).

2) Take advantage of RQ calculations already conducted for single substance risk assessment and omit additional, mathematically equivalent calculation of ETR $_{\text {mix. }}$ by applying $\mathrm{RQ}_{\text {mix }}$ calculations more broadly than currently applied in EFSA [14], (AMiRA Step 3, 4 and 5).

3) Use of screening options to focus the mixture risk assessment on most critical eco-endpoints and exposure scenarios by selecting a worst-case risk assessment scenario based on $\mathrm{RQ}_{\text {mix }}$ (AMiRA Step 3).

4) Use of screening option to identify a risk driver in a mixture risk assessment (AMiRA Step 4).

A detailed description for the individual steps in the AMiRA scheme is outlined in the following section.

\section{The implementation of the approach}

AMiRA Step 1: Evaluation of the spray-drift risk assessment. In addition to the proposed CA approach using RQ available from the single substance risk assessment, we advocate to not neglect the information available where measured toxicity data for the product exist. However, while EFSA [14] includes these data via a time-consuming decision-making process comparing measured toxicity and CA calculated toxicity, we propose to use the measured toxicity data in a simple and straightforward risk assessment with product endpoints and product exposure concentrations after spray-drift entry. Spraydrift entry best reflects the immediate exposure situation

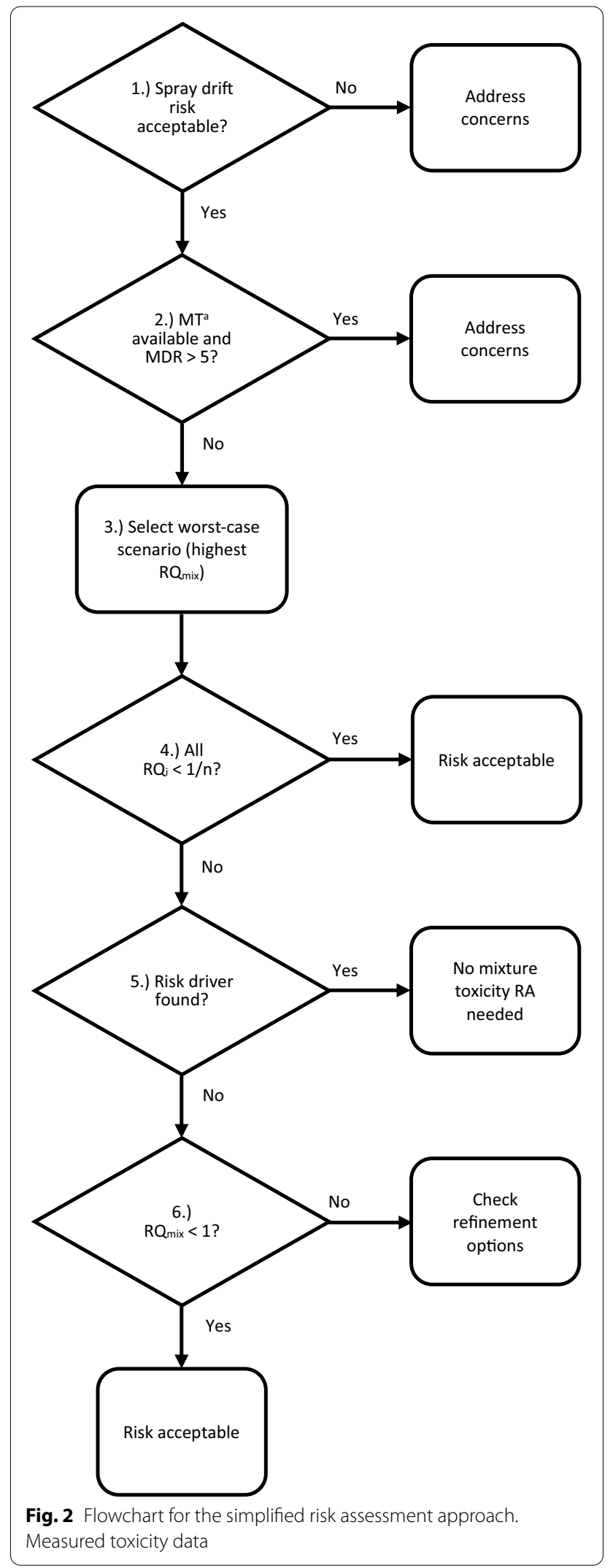


for aquatic organisms to the product as such, while runoff and drainage include soil related processes leading to a fragmentation of the original product and are thus better covered by individual active substance PECs and subsequent $\mathrm{CA}$ estimated mixture risk. For cases, where no measured data are available (e.g. chronic risk after long-term exposure), an assessment can only be based on calculated mixture toxicity. In European risk assessments, spray-drift values from JKI [20] are generally used to describe exposure to the product, however, in cases where other data or models for spray-drift are available or preferred, these can be used as well. In cases where a potential antagonism or synergism has been identified for a product $(\mathrm{MDR}<0.2$ and $>5$ ) this deviation from $C A$ is included in the spray-drift risk assessment.

AMiRA Step 2: Calculation of the MDR to check for major deviations between the calculated and measured mixture toxicity (deviation factor of five is considered to be acceptable). Concerns emerging from major deviations will be addressed by carefully evaluating potential reasons contributing to an apparent synergism or antagonism following the recommendations of EFSA [14]. In all cases, the respective product risk assessment is available. Please note that in case of $M D R<0.2$, the AMiRA approach can be continued without restrictions as it reflects a clear worst-case. If MDR $\leq 5$ reduced complexity regarding the scenario selection can now pragmatically be achieved by selecting the scenario with the worst-case $\mathrm{RQ}_{\text {mix }}$ with corresponding RQ based on PECs from the same FOCUS surface water step still passing the risk assessment (i.e. $\mathrm{RQ}<1$ ).

AMiRA Step 3: Selection of the worst-case scenario (highest $R_{\text {mix }}$ ) per organism group. As sensitivity between organism groups/trophic levels may vary, for the less sensitive organism groups $R Q_{\text {mix }}$ may be found to be $<1$ in this step. It would thus be justified to end the decision tree already here and conclude on a safe use for these groups and continue the decision tree for the sensitive groups only.

AMiRA Step 4: Evaluation of the RQs from $n$ single substances. If all RQs are below $1 / n$, the risk is considered to be acceptable as then the sum of all individual $R Q s\left(=R Q_{\text {mix }}\right)$ will be $<1$. Using $R Q s$ in this approach is justified by an equivalent simplified risk assessment by EFSA ([14], Sect. 10.3.7 in the original publication) using RQs instead of ETRs. Hence, this offers the same level of protection as in EFSA [14]. If at least one RQ exceeds $1 / n$, the risk assessment is continued with the search for a risk driver.

AMiRA Step 5: Evaluation of the existence of a risk driver using PRQs described in a previous section (PRQ calculations for the case study are based on FOCUS surface water step 3 PECs and are provided in the supplemental material). A risk driver is only identified if one substance clearly and consistently drives the risk from the mixture, i.e. if the maximum PRQ from one active substance is at least $90 \%$ (or at most 10\%) for all exposure scenarios, which means that $90 \%$ of the risk (or more) is due to one substance. The RQs for the PRQ calculations have to be based on the same exposure level (e.g. on FOCUS surface water step 3 PECs in the case study) to compare same with same for different substances and correctly reflect the concentration ratios. Important to note is that for this assessment it is not necessary to generate additional PEC values and to advance all mixing partners to the highest FOCUS Step. As the identification of a risk driver is a comparative assessment, it can be based on the RQ values from the lowest available common FOCUS Step (e.g. FOCUS step 2, even if Step 4 is needed for one substance to pass the risk assessment). This also means that RQs used in the comparison may be $>1$ (i.e. not passing the risk assessment at this step), however, this is not important for the question to be answered here (risk driver). If a risk driver can be identified, no further mixture toxicity risk assessment is necessary, and the risk assessment can be based on single substances. Otherwise, the subsequent step must be carried out.

AMiRA Step 6: If no risk driver is identified the mixture risk assessment is continued with the evaluation of the worst-case $\mathrm{RQ}_{\text {mix }}$ corresponding to the worst-case scenario and the worst-case risk assessment, respectively. If the worst-case $R Q_{\text {mix }}$ is below the trigger value of 1 , the risk of the mixture is considered to be acceptable (note that the use of $R Q_{\text {mix }}$ is also proposed in EFSA [14], if the endpoints to be used for the mixture risk assessment refer to the same taxonomic group but are associated with different AFs, and there is no conceptual reason not to apply this approach also when the AF is similar). If the worst-case $R Q_{\text {mix }}$ is above the trigger value of 1 , refinement options (e.g. PEC refinements or consideration of additional risk mitigation for the scenario leading to the worst-case $R_{\text {mix }}$ ) can be considered before repeating the $\mathrm{RQ}_{\text {mix }}$ calculation in the proposed risk assessment scheme.

\section{Illustration of the approach based on a case study}

To illustrate the approach a mixture of the two herbicides flufenacet (FFA) and diflufenican (DFF) is considered. For comparison of the results, also the aquatic mixture toxicity assessment from EFSA [14] was conducted. The used ecotoxicological endpoints, the assumed exposure (PECs from FOCUS scenarios) and detailed calculations from the risk assessment are provided in the Additional file 2: S2. 
Table 3 Spray drift risk assessment based on EFSA [14] and distance-dependent drift values from JKI [20] for the case study with the mixture of FFA and DFF

\begin{tabular}{ll}
\hline Species & Spray drift risk \\
\hline Fish & Acceptable for $1 \mathrm{~m}$ distance from edge-of-field to water body \\
Daphnids & Acceptable for $1 \mathrm{~m}$ distance from edge-of-field to water body \\
Algae & $\begin{array}{l}\text { Acceptable for } 20 \mathrm{~m} \text { distance from edge-of-field to water } \\
\text { body }\end{array}$ \\
Lemna & Acceptable for $5 \mathrm{~m}$ distance from edge-of-field to water body
\end{tabular}

Table 4 Comparison of measured and calculated mixture toxicity using MDR

\begin{tabular}{llll}
\hline Species & $\begin{array}{l}\text { Measured mixture } \\
\text { toxicity (a.s. based) [mg } \\
\text { a.s./L] }\end{array}$ & $\begin{array}{l}\text { Calculated mixture } \\
\text { toxicity [mg a.s./L] }\end{array}$ & MDR \\
\hline Fish & 15.46 & 0.19 & 0.012 \\
Daphnids & 45.8 & 0.1 & 0.002 \\
Algae & 0.001633 & 0.001005 & 0.615 \\
Lemna & 0.01775 & 0.03503 & 1.974 \\
\hline
\end{tabular}

Whenever the risk assessment scheme by EFSA [14] led to the check for apparent antagonism (step 9 in the risk assessment scheme), the measured data were considered to be plausible by default and the risk assessment proceeded by checking the similarity of mixture composition in the product formulation against the $\mathrm{PEC}_{\mathrm{sw}}$ in the FOCUS scenarios (step 3 in the risk assessment scheme). Whenever toxicity or risk drivers were identified, the single substance risk assessments were conducted only considering mitigation measures from FOCUS surface water scenarios (i.e. no RAC refinements or other tools were considered).

A preceding spray-drift risk assessment for the product (see Table 3) indicated an acceptable risk to aquatic organisms from spray-drift exposure for all taxonomic groups. The next step was to conduct a mixture risk assessment using the FOCUS exposure scenarios.

MDR calculations indicated potential antagonism for fish and daphnids (i.e. MDR $<0.2$ ) and moderate deviations for algae and Lemna (i.e. $0.2 \leq \mathrm{MDR} \leq 5$, see Table 4). The potential antagonism (i.e. the measured product toxicity being lower than expected) is most likely explained by the fact that unbound values for the toxicity of DFF in fish and daphnids went into the calculation, i.e. the toxicity for DFF might be even lower than assumed by using the unbound values as bound values practically. This is not further evaluated here, as from a risk assessment perspective, this is of no concern. The risk assessment schemes by EFSA [14] and AMiRA were both unaffected by the MDR outcome and continued with their proceedings as the measured mixture toxicity data were considered to be plausible for the EFSA [14] approach. The comparison of measured and calculated toxicity in Table 4 demonstrates the conservativeness of AMiRA approach to always proceed with calculated toxicity in case of indicated antagonism $(\mathrm{MDR}<0.2)$.

Overall, the results from both approaches are very similar to each other (see Table 5). The risk assessments for daphnids (acute) and algae (chronic) are identical.

Table 5 Risk assessment and RQ comparison for the approach by EFSA [14] and AMiRA

\begin{tabular}{|c|c|c|}
\hline Species & Risk according to EFSA [14] & Risk according to AMiRA \\
\hline Fish & $\begin{array}{l}\text { Acceptable, FOCUS step } 3 \\
\text { FOCUS step 3: } \\
\mathrm{RQ}_{\text {a.s. }}=0.8269<1 \\
\text { (based on single a.s. risk assessment) }\end{array}$ & $\begin{array}{l}\text { Acceptable, FOCUS step } 4 \text { (5 m spray buffer) } \\
\text { FOCUS step 3: } \\
\mathrm{RQ}_{\text {mix }}=1.0012>1 \\
\mathrm{FOCUS} \mathrm{step} 4(5 \mathrm{~m}) \\
\mathrm{RQ}_{\text {mix }}=0.6502<1\end{array}$ \\
\hline Daphnids & $\begin{array}{l}\text { Acceptable, FOCUS step } 4 \text { (10 m spray buffer }+10 \text { m veg. strip) } \\
\text { FOCUS step } 4(10 m+10 \mathrm{~m}): \\
\mathrm{RQ}_{\text {a.s. }}=0.5857<1 \\
\text { (based on single a.s. risk assessment) }\end{array}$ & $\begin{array}{l}\text { Acceptable, FOCUS step } 4 \text { (10 m spray buffer }+10 \text { m veg. strip) } \\
\text { FOCUS step } 4(10 \mathrm{~m}+10 \mathrm{~m}): \\
\mathrm{RQ}_{\text {mix }}=0.5887<1\end{array}$ \\
\hline Algae & $\begin{array}{l}\text { Not acceptable, FOCUS step } 4 \text { (all buffer mitigations) } \\
\text { FOCUS step } 4 \text { (all mitig.): } \\
R_{\text {mix }}=5.9583>1^{a} \\
\text { (based on measured mixture toxicity) }\end{array}$ & $\begin{array}{l}\text { Not acceptable, FOCUS step } 4 \text { (all buffer mitigations) } \\
\text { FOCUS step } 4 \text { (all mitig.): } \\
\mathrm{RQ}_{\text {mix }}=4.5831>1^{\mathrm{a}}\end{array}$ \\
\hline Lemna & $\begin{array}{l}\text { Acceptable, FOCUS step } 4 \text { ( } 20 \text { m spray buffer }+20 \text { m veg. strip) } \\
\text { FOCUS step } 4(10 m+10 \text { m): } \\
R Q_{\text {mix }}=1.0379>1 \\
\text { FOCUS step } 4(20 m+20 \mathrm{~m}): \\
R_{\text {mix }}=0.5438<1 \\
\text { (based on measured mixture toxicity) }\end{array}$ & $\begin{array}{l}\text { Acceptable, FOCUS step } 4 \text { (10 m spray buffer }+10 \text { m veg. strip) } \\
\text { FOCUS step } 4(10 \mathrm{~m}+10 \mathrm{~m}) \text { : } \\
\mathrm{RQ}_{\text {mix }}=0.5693<1\end{array}$ \\
\hline
\end{tabular}

a Please note, that necessarily all $R_{\text {mix }}>2$ (in case of two active substances in the mixture) consist of at least one single substance $R Q>1$ leading to not acceptable risk even for a single substance risk assessment 
The risk assessment for fish (acute) is more conservative using AMiRA instead of following EFSA [14]. The less conservative results from the risk assessment based on EFSA [14] is due to two reasons:

1) Measured mixture toxicity data indicating strong antagonism $(\mathrm{MDR}<0.2)$ were used for four of nine exposure scenarios leading to acceptable risk (FOCUS step 3).

2) A toxicity driver was identified for five of nine exposure scenarios leading to acceptable risk (FOCUS step 3) for all single substance risk assessments.

The identification of a toxicity driver (based on the approach by EFSA [14]) revealed its conceptual weakness to not take the environmental exposure into account, but being based on concentrations in the product. The identification of a toxicity driver and following conclusion of single substance assessment being protective led to different conclusions for EFSA [14] and AMiRA. This is especially important for $R Q_{\text {mix }}$ close to 1 , where minor differences in individual $R Q$ can result in $R Q_{\text {mix }}$ just below/above the trigger (in this case: $R_{\text {mix }}=1.0012$ (not acceptable, based on calculated mixture toxicity) or $R Q=0.8269$ (single substance RQ identified as toxicity driver, acceptable risk) In contrast to the toxicity driver evaluation according to EFSA [14] when interpreting the composition as "concentrations in product", the risk driver evaluation from AMiRA showed no evidence for a consistent risk driver, i.e. not all PRQs exceeded 90\% (the scenario-specific PRQs ranged from 45 to $96 \%$ ). Notably, no risk driver was identified for the worst-case scenario for the risk assessment (i.e. the scenario with the highest $\left.\mathrm{RQ}_{\text {mix }}\right)$ selected at FOCUS step $3(\mathrm{PRQ}=83 \%)$. Following EFSA [14] the risk assessment based on single substances neglected up to $55 \%$ of the risk from the substance that was consequently then not further considered in the assessment of mixture risk.

The risk assessment for Lemna (chronic) with AMiRA is less conservative than the approach by EFSA [14], since the risk from EFSA [14] is assessed based on measured mixture toxicity data: The MDR $>1$ indicates that the measured mixture toxicity is higher than the calculated mixture toxicity leading to a 'borderline' situation for the
$\mathrm{RQ}_{\text {mix }} \sim 1$ based on measured mixture toxicity at FOCUS step 4 (see RQ values in Table 5). Overviews of the results from the risk assessment conducted with the simplified AMiRA approach and the risk assessment following EFSA [14] are shown in Tables 5, 6 and 7.

\section{Conclusion from the case study}

The results showed that in some cases results from both approaches differ to some extent. These differences were due to the use of measured data and questionable toxicity driver identifications in the EFSA [14] scheme. When the risk assessment was based on measured mixture toxicity data and the calculated MDR was greater than 1 for Lemna (i.e. the measured mixture toxicity was higher than the calculated mixture toxicity), the approach by EFSA [14] resulted in a more conservative assessment. If the MDR is below 1 (i.e. the measured mixture toxicity is lower than the calculated mixture toxicity), the risk assessment based on measured mixture toxicity could result in a less conservative assessment than the proposed simplified AMiRA approach.

When the risk assessment was based on single substance risk assessment due to the questionable identification of a toxicity driver regarding scenarios for fish, the approach by EFSA [14] resulted in a less conservative assessment than the AMiRA approach. However, it must be noted that the result would have been the same for fish if the basic assumption in the case of apparent antagonism (EFSA [14], Step 9) had been that the measured data were not plausible (i.e. calculated toxicity would have been used instead of going to Step 3).

In many cases, the risk assessments based on AMiRA and the approach by EFSA [14] will be identical. Causes for differences can be identified at specific points, namely the use of measured data (e.g. if the measured data indicates strong synergism, i.e. MDR $>5$, or if the measured data are similar to the calculated data, i.e. the MDR is around 1 , and RQ values close to 1 cause a 'borderline' situation for the risk assessment) or the identification of toxicity drivers. The approaches are identical if:

1) measured data are not available and there is no evidence for synergism or

Table 6 Risk assessment results according to AMiRA

\begin{tabular}{llll}
\hline Species & Step in flowchart & Risk assessment based on & Risk and corresponding FOCUS step \\
\hline Fish & $1->2->3->4->5->6$ & Calculated mixture toxicity & Acceptable, FOCUS step 4 (5 m spray buffer) \\
Daphnids & $1->2->3->4->5$ & Single a.s. risk assessment & Acceptable, FOCUS step 4 (10 m spray buffer + 10 m veg. strip) \\
Algae & $1->2->3->4->5->6$ & Calculated mixture toxicity & Not acceptable, FOCUS step 4 (all buffer mitigations) \\
Lemna & $1->2->3->4->5->6$ & Calculated mixture toxicity & Acceptable, FOCUS step 4 (10 m spray buffer + 10 m veg. strip) \\
\hline
\end{tabular}


Table 7 Risk assessment results from EFSA [14] approach

\begin{tabular}{|c|c|c|c|c|}
\hline Species & Scenario & Step in flowchart & Risk assessment based on & Risk per scenario and corresponding FOCUS step \\
\hline \multirow[t]{9}{*}{ Fish } & D3, ditch & $1->2->9->3->4$ & Measured mixture toxicity & Acceptable, FOCUS step 3 \\
\hline & D4, pond & $1->2->9->3->5->6$ & Single a.s. risk assessment & Acceptable, FOCUS step 3 \\
\hline & D4, stream & $1->2->9->3->4$ & Measured mixture toxicity & Acceptable, FOCUS step 3 \\
\hline & D5, pond & $1->2->9->3->5->6$ & Single a.s. risk assessment & Acceptable, FOCUS step 3 \\
\hline & D5, stream & $1->2->9->3->4$ & Measured mixture toxicity & Acceptable, FOCUS step 3 \\
\hline & R1, pond & $1->2->9->3->4$ & Measured mixture toxicity & Acceptable, FOCUS step 3 \\
\hline & R1, stream & $1->2->9->3->5->6$ & Single a.s. risk assessment & Acceptable, FOCUS step 3 \\
\hline & R3, stream & $1->2->9->3->5->6$ & Single a.s. risk assessment & Acceptable, FOCUS step 3 \\
\hline & R4, stream & $1->2->9->3->5->6$ & Single a.s. risk assessment & Acceptable, FOCUS step 3 \\
\hline \multirow[t]{9}{*}{ Daphnids } & D3, ditch & $1->2->9->3->4$ & Measured mixture toxicity & Acceptable, FOCUS step 3 \\
\hline & D4, pond & $1->2->9->3->5->6$ & Single a.s. risk assessment & Acceptable, FOCUS step 3 \\
\hline & D4, stream & $1->2->9->3->4$ & Measured mixture toxicity & Acceptable, FOCUS step 3 \\
\hline & D5, pond & $1->2->9->3->5->6$ & Single a.s. risk assessment & Acceptable, FOCUS step 3 \\
\hline & D5, stream & $1->2->9->3->4$ & Measured mixture toxicity & Acceptable, FOCUS step 3 \\
\hline & R1, pond & $1->2->9->3->4$ & Measured mixture toxicity & Acceptable, FOCUS step 3 \\
\hline & R1, stream & $1->2->9->3->5->6$ & Single a.s. risk assessment & Acceptable, FOCUS step 4 (5 m spray buffer) \\
\hline & R3, stream & $1->2->9->3->5->6$ & Single a.s. risk assessment & Acceptable, FOCUS step 4 (5 m spray buffer) \\
\hline & R4, stream & $1->2->9->3->5->6$ & Single a.s. risk assessment & Acceptable, FOCUS step 4 (10 m spray buffer +10 m veg. strip) \\
\hline \multirow[t]{9}{*}{ Algae } & D3, ditch & $1->2->3->4$ & Measured mixture toxicity & Acceptable, FOCUS step 4 (20 m spray buffer) \\
\hline & D4, pond & $1->2->3->5->8$ & Calculated mixture toxicity & Not acceptable, FOCUS step 4 (all buffer mitigations) \\
\hline & D4, stream & $1->2->3->4$ & Measured mixture toxicity & Not acceptable, FOCUS step 4 (all buffer mitigations) \\
\hline & D5, pond & $1->2->3->5->8$ & Calculated mixture toxicity & Not acceptable, FOCUS step 4 (all buffer mitigations) \\
\hline & D5, stream & $1->2->3->4$ & Measured mixture toxicity & Not acceptable, FOCUS step 4 (all buffer mitigations) \\
\hline & R1, pond & $1->2->3->4$ & Measured mixture toxicity & Acceptable, FOCUS step 3 \\
\hline & R1, stream & $1->2->3->5->8$ & Calculated mixture toxicity & Not acceptable, FOCUS step 4 (all buffer mitigations) \\
\hline & R3, stream & $1->2->3->5->8$ & Calculated mixture toxicity & Not acceptable, FOCUS step 4 (all buffer mitigations) \\
\hline & R4, stream & $1->2->3->5->8$ & Calculated mixture toxicity & Not acceptable, FOCUS step 4 (all buffer mitigations) \\
\hline \multirow[t]{9}{*}{ Lemna } & D3, ditch & $1->2->3->4$ & Measured mixture toxicity & Acceptable, FOCUS step 3 \\
\hline & D4, pond & $1->2->3->4$ & Measured mixture toxicity & Acceptable, FOCUS step 3 \\
\hline & D4, stream & $1->2->3->4$ & Measured mixture toxicity & Acceptable, FOCUS step 3 \\
\hline & D5, pond & $1->2->3->4$ & Measured mixture toxicity & Acceptable, FOCUS step 3 \\
\hline & D5, stream & $1->2->3->4$ & Measured mixture toxicity & Acceptable, FOCUS step 3 \\
\hline & R1, pond & $1->2->3->4$ & Measured mixture toxicity & Acceptable, FOCUS step 3 \\
\hline & R1, stream & $1->2->3->4$ & Measured mixture toxicity & Acceptable, FOCUS step 4 (10 m spray buffer +10 m veg. strip) \\
\hline & R3, stream & $1->2->3->4$ & Measured mixture toxicity & Acceptable, FOCUS step 4 (20 m spray buffer +20 m veg. strip) \\
\hline & R4, stream & $1->2->3->4$ & Measured mixture toxicity & Acceptable, FOCUS step 4 (10 m spray buffer +10 m veg. strip) \\
\hline
\end{tabular}

2) the measured data indicates strong antagonism $(\mathrm{MDR}<0.2)$ and is considered not to be plausible or

3) the measured mixture toxicity data from toxicological studies is not comparable to the mixture composition under field conditions and there is no evidence for a toxicity driver.

\section{Discussion}

We here propose a simplified scheme (AMiRA) to assess aquatic mixture risk based on the following key points contributing to efficiency gain:

- Take advantage of RQ calculations already available from single substance risk assessment to conduct a mixture risk assessment based on CA.

- Take advantage of empirically measured product toxicity data in a product risk assessment for spray-drift entry. 
- Include an assessment to determine a worst-case scenario and apply a risk envelope approach focussing on the critical areas of an assessment.

- Include an assessment of risk drivers to identify scenarios where the active substance risk assessment covers mixture risk assessment.

- Align assessment metrics for single substance and mixture risk assessment ( $R Q$ and $R Q_{\text {mix }}$ ) to enable efficient comparison of single substance and mixture risk assessment and thereby decrease hurdles for communication of risk assessment outcomes and increase transparency.

For the risk assessment mixture toxicity approaches have to take various aspects into account, such as the selection of a suitable exposure scenario, the search for a toxicity or risk driver, or the proper calculation of mixture toxicity. EFSA [14] has presented a first regulatory approach which integrates all of these. While this approach is rather complex and may still be optimised, it offers a first valuable tool for the risk assessment. In the present paper, a new, more efficient aquatic risk assessment scheme (AMiRA) is proposed as an alternative pragmatic approach, which offers a comparable level of protection despite of a much lower complexity, and which could be used to quickly identify both non-critical areas of an assessment of combined toxicity as well as focus the evaluation to areas that are driving the overall conclusion for risk management. Chen et al. [8] is considered a valuable extension to EFSA [14]. Therefore, the improvements of the proposed AMiRA approach over the approach by EFSA [14] also partially apply to Chen et al. [8]. The overly conservative construction of a more than worst-case exposure scenario by Chen et al. [8] goes beyond the selection of a worst-case scenario in the AMiRA approach, but makes it impossible to identify a toxicity or risk driver from a realistic exposure scenario.

One of the key advantages of AMiRA is to make use of calculations of RQs already available from the single substance risk assessment for further evaluation of mixture risk. Based on the 6-8 ecological endpoints and up to 252 exposure scenarios to be assessed per endpoint, a maximum of $2016 \mathrm{PEC}_{\text {mix }}, \mathrm{EC}_{\mathrm{xmix}}$ and/or ETR $\mathrm{Emix}_{\text {values need }}$ to be calculated, assessed and evaluated in subsequent steps in the EFSA decision tree. Thus, relying on $R_{\text {mix }}$ can result in substantial resource savings while keeping the same information for mixture risk evaluation.

For illustration of the new approach, a mixture risk assessment has been conducted for a formulation containing two substances. The results from this case study reveal that the proposed risk assessment scheme has the same level of protection as EFSA [14] when the risk assessment is based on calculated mixture toxicity (=in cases composition of the product and environmental compositions differ and there are no indications of synergism. In fact, in this case both approaches are mathematically equivalent. Choosing worst-case $R_{Q_{\text {mix }}}$ from exposure scenarios in the first place always leads to worst-case risk for calculated mixture toxicity. Based on evaluations of comparability between measured and predicted toxicity (MDR), for MDR between 1 and 5, EFSA [14] was more conservative (= risk assessment based on measured mixture toxicity data and the measured toxicity is higher than the calculated one) while for MDR between 0.2 and 1, AMiRA resulted in higher conservatism. However, this level of conservativeness is limited to the MDR deviation factor of five that is considered to be acceptable to account for biological and other variation in testing [14]. The proposed AMiRA approach includes the use of measured toxicity data in a direct spray-drift entry based risk assessment, using measured data in all cases and thus accounting for any higher-than-calculated toxicity in the conclusion. Therefore, results from AMiRA can still be considered reliable.

EFSA [14] is not fully clear regarding the determination of a driving substance and depending on the composition included in the calculation (product or environment) either a risk or toxicity driver is identified. When considered product composition and thus identification of a toxicity driver in the approach by EFSA [14] we could show the conceptual weaknesses in our case study. The illustrated case study revealed that the toxicity driver approach (which identified a toxicity driving substance and concluded that the risk is covered by a single substance risk assessment) led to a neglect of substantial additional risk from the other substances in the mixture product which then were not covered by the single substance risk assessment. That can be avoided by searching for a risk driver as proposed in the AMiRA approach and/or providing additional specification in EFSA [14].

If the measured data show lower toxicity than the calculated data (i.e. $M D R<1$ ), the risk assessment based on measured mixture toxicity could result in a less conservative assessment than the proposed simplified approach. In practice, the use of measured data may often be limited, since the calculated proportions of the substances in the PEC values of some scenarios will differ from those in the formulation toxicity studies. This is particularly frequent in the drainage and pond scenarios, as for these substance properties are especially relevant. Hence, in practice the use of measured data may be limited to a product risk assessment with exposure via spray-drift.

As there is ongoing research activity in the EU considering combined exposure to multiple chemicals [6], future mixture toxicity studies and their evaluation will 
contribute to the improvement of the proposed simplified approach.

Overall, it was shown that the proposed approach yields substantial gains in simplification and efficiency when assessing mixture toxicity while achieving comparable protection levels. By using RQs, the results can be interpreted quickly, lowering the hurdles to communicating risk assessment conduct and outcome and the translation into risk management activities.

\begin{abstract}
Abbreviations
AF: Assessment factor; AMiRA: Alternative approach for Aquatic Mixture Risk Assessment; a.s.: Active substance; CA: Concentration addition; DFF: Diflufenican; ECx: Concentration showing $x \%$ effect; ETR: Exposure toxicity ratio; FFA: Flufenacet; IA: Independent action; MDR: Model deviation ratio; MoA: Mode of action; PEC: Predicted environmental concentration; PNEC: Predicated no-effect concentration; PPP: Plant protection product; PRQ: Proportional risk quotient; RAC: Regulatory acceptable concentration; RQ: Risk quotient; TS: Toxicological similarity; TU: Toxic unit.
\end{abstract}

\section{Supplementary Information}

The online version contains supplementary material available at https://doi. org/10.1186/s12302-022-00594-3.

Additional file 1. Supplemental material S1 - Background information on the key questions for the development of an alternative mixture risk assessment approach.

Additional file 2. Supplemental material S2 - Case study information.

\section{Acknowledgements}

Not applicable.

\section{Authors' contributions}

All authors contributed equally to the writing of the document. All authors read and approved the final manuscript.

\section{Funding}

The present study was funded by Bayer AG.

\section{Availability of data and materials}

The datasets for the case study of this article are included within the supplemental material.

\section{Declarations}

Ethics approval and consent to participate Not applicable.

\section{Consent for publication}

Not applicable.

\section{Competing interests}

AG and ME are employed at Bayer AG and work for the registration of plant protection products. CD and MW work as contractors for Bayer AG. The authors declare no other competing interests.

\section{Author details}

${ }^{1}$ WSC Scientific GmbH, Carl-Benz-Str. 1, 69115 Heidelberg, Germany. ${ }^{2}$ Bayer AG, Crop Science Division, Alfred-Nobel-Str. 50, 40789 Monheim am Rhein, Germany,
Received: 20 September 2021 Accepted: 28 January 2022

Published online: 19 February 2022

\section{References}

1. AGES (2018) Working document of the Central Zone in the authorisation of plant protection products-Section 8-Environmental fate and behaviour, Version 1 rev. 1. https://circabc.europa.eu/d/a/workspace/ SpacesStore/e4dac049-ae0e-4ea6-9dca-e8a7ef5e81c0/Working\%20doc ument $\% 20$ of $\% 20$ the $\% 20$ central\%20zone\%20Environmental\%20Fate\% 20-\%20Ver\%201.1(0).docx. Accessed 10 Dec. 2020.

2. Altenburger R, Arrhenius A, Backhaus T, Coors A, Faust M, Zitzkat D (2013) Ecotoxicological combined effects from chemical mixtures_-Part 1: Relevance and adequate consideration in environmental risk assessment of plant protection products and biocides. Project No. (FKZ) 370965404. Umweltbundesamt, Dessau-Rosslau, Germany.

3. Backhaus T, Faust M (2012) Predictive environmental risk assessment of chemical mixtures: a conceptual framework. Environ Sci Technol 46:2564-2573. https://doi.org/10.1021/es2034125

4. Backhaus T, Altenburger R, Faust M, Frein D, Frische T, Johansson P, Kehrer A, Porsbring T (2013) Proposal for environmental mixture risk assessment in the context of the biocidal product authorization in the EU. Environ Sci Eur. https://doi.org/10.1186/2190-4715-25-4

5. Belden JB, Gilliom RJ, Lydy MJ (2007) How well can we predict the toxicity of pesticide mixtures to aquatic life? Integr Environ Assess Manag 3:364-372. https://doi.org/10.1002/ieam.5630030307

6. Bopp SK, Barouki R, Brack W, Dalla Costa S, Dorne JCM, Drakvik PE, Faust M, Karjalainen TK, Kephalopoulos S, van Klaveren J, Kolossa-Gehring M, Kortenkamp A, Lebret E, Lettieri T, Nørager S, Rüegg J, Tarazona JV, Trier X, van de Water B, van Gils J, Bergman Å (2018) Current EU research activities on combined exposure to multiple chemicals. Environ Int 120:544562. https://doi.org/10.1016/j.envint.2018.07.037

7. Brown VM (1968) The calculation of the acute toxicity of mixtures of poisons to rainbow trout. Wat Res 2:723-733. https://doi.org/10.1016/ 0043-1354(68)90044-4

8. Chen L, Li S, Zhou Y, Zhou X, Jiang H, Liu X, Yuan S (2020) Risk assessment for pesticide mixtures on aquatic ecosystems in China: a proposed framework. Pest Manag Sci 76:444-453. https://doi.org/10.1002/ps.5529

9. Danish EPA (2021) Aquatic MixTox tool. https://eng.mst.dk/media/ 220195/agd_aquamix_v115.xlsm. Accessed 28 Jul 2021

10. Ebeling, M (2013) Chemical mixtures: integrating environmental exposure and effect assessment. Conference: Informa Life Sciences, AgChem Forum, Barcelona

11. EFSA (European Food Safety Authority) (2008) Scientific Opinion of the Panel on Plant Protection Products and their Residues (PPR Panel) on a request from the EFSA evaluate the suitability of existing methodologies and if appropriate, the identification of new approaches to assess cumulative and synergistic risks from pesticides to human health with a view to set MRLs for those pesticides in the frame of Regulation (EC) 396/2005. EFSA J 6:705. https://doi.org/10.2903/j.efsa.2008.705

12. EFSA (European Food Safety Authority) (2009) Guidance document on risk assessment for birds \& mammals on request from EFSA. EFSA J 7:1438. https://doi.org/10.2903/j.efsa.2009.1438

13. EFSA (EFSA Panel on Plant Protection Products and their Residues) (2012) Scientific opinion on the science behind the development of a Risk Assessment of Plant Protection Products on bees (Apis mellifera, Bombus spp. and solitary bees). EFSA J 10:2668. https://doi.org/10.2903/j.efsa. 2012.2668

14. EFSA (EFSA Panel on Plant Protection Products and their Residues) (2013) Guidance on tiered risk assessment for plant protection products for aquatic organisms in edge-of-field surface waters. EFSA J 11:3290. https://doi.org/10.2903/j.efsa.2013.3290

15. Finizio A, Villa S, Vighi M (2005) Predicting pesticide mixtures load in surface waters from a given crop. Agr Ecosys Env 111:111-118. https:// doi.org/10.1016/j.agee.2005.05.009

16. FOCUS (2001) FOCUS Surface Water Scenarios in the EU Evaluation Process under 91/414/EEC. Report of the FOCUS Working Group on Surface Water Scenarios, EC Document Reference SANCO/4802/2001-rev.2. 245 $\mathrm{pp}$ 
17. FPS Health, Food Chain Safety and Environment for Belgium (2021) Calculation tool: Mixture toxicity (Belgium, v.2). https://fytoweb.be/en/ guide/crop-protection/guidance-ecotoxicology. Accessed 26 Nov 2021

18. Frische T, Matezki S, Wogram J (2014) Environmental risk assessment of pesticide mixtures under regulation 1107/2009/EC: a regulatory review by the German Federal Environment Agency (UBA). J Verbr Lebensm 9:377-389. https://doi.org/10.1007/s00003-014-0916-6

19. Hillebrand M, Duquesne S, Aagard A, Kraemer W, Hauge Skarsjo M, Zorn M, Pedersen S, Trobej M, Gassner-Schneckenleithner C, Egerer S, Frishe T (2021) Aquatic mixture toxicity tool and additional information (version 1.15). Zenodo. https://doi.org/10.5281/zenodo.4593676.

20. JKI (2021) Abdrifteckwerte - Tabelle der Abdrifteckwerte. https://www. julius-kuehn.de/media/Institute/AT/PDF_RichtlinienListenPruefberichte/ Abdrifteckwerte/Tabelle_der_Abdrifteckwerte.xls. Accessed 5 May 2021.

21. Junghans M, Backhaus T, Faust M, Scholze M, Grimme LH (2006) Application and validation of approaches for the predictive hazard assessment of realistic pesticide mixtures. Aqua Toxicol 76:93-110. https://doi.org/10. 1016/j.aquatox.2005.10.001

22. Kortenkamp A, Backhaus T, Faust M (2009) State of the art report on mixture toxicity. Final report, study Contract number 070307/2007/485103/ ETU/D.1. European Commission, Brussels, Belgium

23. Kortenkamp A, Ermler S, Scholze M, Faust M, Backhaus T, Posthuma L, de Zwart D, Focks A, Baveco H, van Gils J (2018) Solutions for present and future emerging pollutants in land and water resources management. Common assessment framework for HRA and ERA higher tier assessments including fish and drinking water and multi-species ERA via SSD, population-level ERA via IBM and food web vulnerability ERA. https:// www.solutions-project.eu/wp-content/uploads/2018/11/D18.1_SOLUT IONS-D18_1-after-peer-review-clean-V2_Kortenkamp_chm_with_annex. pdf. Accessed 21 Oct 2020

24. Meek ME, Boobis AR, Crofton KM, Heinemeyer G, Raaij MV, Vickers C (2011) Risk assessment of combined exposure to multiple chemicals: a WHO/IPCS framework. Regul Toxicol Pharmacol. https://doi.org/10.1016/j. yrtph.2011.03.010

25. OECD (Organisation for Economic Co-operation and Development) (2000). Guidance document on aquatic toxicity testing of difficult substances and mixtures. Series on Testing and Assessment 23. ENV/JM/ MONO(2000)6

26. Price $P, \operatorname{Han} X$ (2011) Maximum cumulative ratio (MCR) as a tool for assessing the value of performing a cumulative risk assessment. Int J Environ Res Public Health 8:2212-2225. https://doi.org/10.3390/ijerp h8062212

27. Price P, Dhein E, Hamer M, Han X, Heneweer M, Junghans M, Kunz P, Magyar C, Rodriguez PH (2012) A decision tree for assessing effects from exposures to multiple substances. Environ Sci Eur. https://doi.org/10. 1186/2190-4715-24-26

28. SCHER (Scientific Committee on Health and Environmental Risks), SCCS (Scientific Committee on Consumer Safety), SCENIHR (Scientific Committee on Emerging and Newly Identified Health Risks) (2012) Opinion on the Toxicity and Assessment of Chemical Mixtures. European Union. https://doi.org/10.2772/21444

29. US EPA (US Environmental Protection Agency) (2000) Supplementary guidance for conducting health risk assessment of chemical mixtures. http://www.oecd.org/officialdocuments/publicdisplaydocumentpdf/? cote $=E N V / J M / M O N O(2000) 6 \&$ docLanguage $=E n$. Accessed 21 Oct. 2020.

30. Verro R, Finizio A, Otto S, Vighi M (2009) Predicting pesticide environmental risk in intensive agricultural areas. ii-screening level risk assessment of complex mixtures in surface waters. Environ Sci Technol 43:530-537. https://doi.org/10.1021/es801858h

31. Weyers A, Bender K, Ebeling M, Gladbach A (2018) Simplify: Reasonable approaches to Mixtox assessment for plant protection products. Conference: SETAC Europe 28th Annual Meeting, Rome. https://doi.org/10. 13140/RG.2.2.31259.92960.

\section{Publisher's Note}

Springer Nature remains neutral with regard to jurisdictional claims in published maps and institutional affiliations.

\section{Submit your manuscript to a SpringerOpen ${ }^{\circ}$ journal and benefit from:}

- Convenient online submission

- Rigorous peer review

- Open access: articles freely available online

- High visibility within the field

- Retaining the copyright to your article

Submit your next manuscript at $\boldsymbol{\nabla}$ springeropen.com 